\title{
ON THE ABSOLUTE SUMMABILITY FACTORS OF TYPE $(A, B)$
}

\author{
HIKMET SEYHAN
} \begin{abstract}
In this paper we establish a relation between the $\varphi-\left|\bar{N}, p_{n} ; \delta\right|_{k}$ and $\psi-\left|\bar{N}, q_{n} ; \delta\right|_{k}$
summability methods, which generalizes a result of Mishra [2].
\end{abstract}
\section{Introduction}

Let $\left(\varphi_{n}\right)$ be a sequence of positive real numbers and let $\sum a_{n}$ be a given infinite series with the sequence of partial sums $\left(s_{n}\right)$. Let $\left(p_{n}\right)$ be a sequence of positive real numbers

$$
P_{n}=\sum_{v=0}^{n} p_{v} \rightarrow \infty \text { as } n \rightarrow \infty, \quad\left(P_{-i}=p_{-i}=0, i \geq 1\right) .
$$

The sequence-to-sequence transformation

$$
t_{n}=\frac{1}{P_{n}} \sum_{v=0}^{n} p_{v} s_{v}
$$

defines the sequence $\left(t_{n}\right)$ of the $\left(\bar{N}, p_{n}\right)$ means of the sequence $\left(s_{n}\right)$, generated by the sequence of coefficients $\left(p_{n}\right)$.

The series $\sum a_{n}$ is said to be summable $\left|\bar{N}, p_{n}\right|_{k}, k \geq 1$, if (see [1])

$$
\sum_{n=1}^{\infty}\left(P_{n} / p_{n}\right)^{k-1}\left|t_{n}-t_{n-1}\right|^{k}<\infty
$$

and it is said to be summable $\varphi-\left|\bar{N}, p_{n} ; \delta\right|_{k}, k \geq 1$ and $\delta \geq 0$, if (see [3])

$$
\sum_{n=1}^{\infty} \varphi_{n}^{\delta k+k-1}\left|t_{n}-t_{n-1}\right|^{k}<\infty .
$$

If we take $\delta=0$ and $\varphi_{n}=\frac{P_{n}}{p_{n}}$, then $\varphi-\left|\bar{N}, p_{n} ; \delta\right|_{k}$ summability is the same as $\left|\bar{N}, p_{n}\right|_{k}$
summability.

Received June 12, 1998.

1991 Mathematics Subject Classification. 40D15, 40F05, 40G99.

Key words and phrases. Absolute summability, summability factors, infinite series. 
If $\sum a_{n} \lambda_{n}$ is summable by a method $B$ whenever $\sum a_{n}$ is summable by a method $A$, then we say that the factor $\lambda_{n}$ is of type $(A, B)$ and write

$$
\lambda_{n} \in(A, B) .
$$

\section{The Following Theorem Is Known}

Theorem A.([2]) Let the sequences $\left(p_{n}\right)$ and $\left(q_{n}\right)$ be such that $p_{n}>0, q_{n}>0$, $P_{n} \rightarrow \infty, Q_{n} \rightarrow \infty$ and

$$
P_{n} / p_{n}=O\left(Q_{n} / q_{n}\right)
$$

Then in order that

$$
\lambda_{n} \in\left(\left|\bar{N}, q_{n}\right|_{k},\left|\bar{N}, p_{n},\right|_{k}\right), \quad k \geq 1
$$

it is sufficient that

$$
\lambda_{n}=O\left(q_{n} P_{n} / p_{n} Q_{n}\right)
$$

and

$$
P_{n} Q_{n-1} \Delta \lambda_{n}+\left(q_{n} P_{n}-p_{n} Q_{n}\right) \lambda_{n}=O\left(q_{n} P_{n}\right),
$$

where $\Delta \lambda_{n}=\lambda_{n}-\lambda_{n+1}$.

3. The Object of This Paper is to Generalize Above Theorem in the Following Form

Theorem. Let $k \geq 1$ and $0 \leq \delta<1 / k$. Let $\left(\varphi_{n}\right)$ and $\left(\psi_{n}\right)$ be sequences of positive numbers such that

$$
\varphi_{n}=O\left(\psi_{n}\right) .
$$

Let the sequences $\left(p_{n}\right)$ and $\left(q_{n}\right)$ be such that $p_{n}>0, q_{n}>0, P_{n} \rightarrow \infty, Q_{n} \rightarrow \infty$ and (6) is satisfied. If

$$
\sum_{n=v+1}^{\infty} \frac{\varphi_{n}^{\delta k+k-1} p_{n}^{k}}{P_{n}^{k} P_{n-1}}=O\left\{\frac{\varphi_{v}^{\delta k+k-1} p_{v}^{k-1}}{P_{v}^{k}}\right\}
$$

then in order that

$$
\lambda_{n} \in\left(\psi-\left|\bar{N}, q_{n} ; \delta\right|_{k}, \varphi-\left|\bar{N}, p_{n} ; \delta\right|_{k}\right),
$$

it is sufficient that the conditions (8) and (9) hold.

It may be remarked that, in this thorem, if we take $\delta=0, \varphi_{n}=\frac{P_{n}}{p_{n}}$ for $\varphi-\left|\bar{N}, p_{n} ; \delta\right|_{k}$ and $\delta=0, \psi_{n}=\frac{Q_{n}}{q_{n}}$ for $\psi-\left|\bar{N}, q_{n} ; \delta\right|_{k}$, then we get Theorem A. In this case condition (11) reduces to

$$
\sum_{n=v+1}^{\infty} \frac{p_{n}}{P_{n} P_{n-1}}=O\left(\frac{1}{P_{v}}\right)
$$

which always holds. 


\section{Proof of the Theorem}

Let

$$
T_{n}=\frac{1}{Q_{n}} \sum_{v=0}^{n} q_{v} s_{v}=\frac{1}{Q_{n}} \sum_{v=0}^{n}\left(Q_{n}-Q_{v-1}\right) a_{v} .
$$

Write $T_{n}-T_{n-1}=b_{n}$ (write $T_{-1}=0$ ) so that $T_{n}=b_{0}+b_{1}+b_{2}+\cdots+b_{n}$. Thus we suppose that in seies form the $\left(\bar{N}, q_{n}\right)$ transform of $\sum a_{v}$ is $\sum b_{n}$. In a similar way suppose that in series form the $\left(\bar{N}, p_{n}\right)$ transform of $\sum a_{v} \lambda_{v}$ is $\sum c_{n}$. Now we have for $n \geq 1$

$$
b_{n}=\frac{q_{n}}{Q_{n} Q_{n-1}} \sum_{v=1}^{n} Q_{v-1} a_{v}
$$

which gives

$$
a_{n}=\left(\frac{Q_{n}}{q_{n}}\right) b_{n}-\left(\frac{Q_{n-2}}{q_{n-1}}\right) b_{n-1} .
$$

Replacing $a_{v}$ by $a_{v} \lambda_{v}$ and interchanging $p, P$ with $q, Q$ we have for $n \geq 1$

$$
c_{n}=\frac{p_{n}}{P_{n} P_{n-1}} \sum_{v=1}^{n} P_{v-1} a_{v} \lambda_{v} \text {. }
$$

Substituting (15) in (16), we get

$$
\begin{aligned}
c_{n} & =\frac{p_{n}}{P_{n} P_{n-1}} \sum_{v=1}^{n} P_{v-1} \lambda_{v}\left(\left(Q_{v} / q_{v}\right) b_{v}-\left(Q_{v-2} / q_{v-1}\right) b_{v-1}\right) \\
& =\frac{p_{n}}{P_{n} P_{n-1}} \sum_{v=1}^{n-1} b_{v} / q_{v}\left(P_{v-1} Q_{v} \lambda_{v}-P_{v} Q_{v-1} \lambda_{v+1}\right)+\left(p_{n} Q_{n} / q_{n} P_{n}\right) \lambda_{n} b_{n} \\
& =\frac{p_{n}}{P_{n} P_{n-1}} \sum_{v=1}^{n-1} b_{v} / q_{v}\left(P_{v} Q_{v-1} \Delta \lambda_{v}+\left(q_{v} P_{v}-p_{v} Q_{v}\right) \lambda_{v}\right)+\left(p_{n} Q_{n} / q_{n} P_{n}\right) \lambda_{n} b_{n}
\end{aligned}
$$

Now using (8) and (9) in (17) we have

$$
\begin{aligned}
c_{n} & =O\left(\frac{p_{n}}{P_{n} P_{n-1}} \sum_{v=1}^{n-1} b_{v} P_{v}\right)+O\left(b_{n}\right) \\
& =O\left(C_{n, 1}\right)+O\left(C_{n, 2}\right) .
\end{aligned}
$$

By Hölder's inequality we get

$$
\begin{aligned}
\left\{\sum_{v=1}^{n-1}\left|b_{v}\right| P_{v}\right\}^{k} & \leq \sum_{v=1}^{n-1}\left\{\left|b_{v}\right|^{k} P_{v}^{k} / p_{v}^{k-1}\right\}\left\{\sum_{v=1}^{n-1} p_{v}\right\}^{k-1} \\
& \leq P_{n-1}^{k-1} \sum_{v=1}^{n-1}\left|b_{v}\right|^{k} P_{v}^{k} / p_{v}^{k-1}
\end{aligned}
$$


so that

$$
\begin{aligned}
\sum_{n=1}^{\infty} \varphi_{n}^{\delta k+k-1}\left|C_{n, 1}\right|^{k} & =\sum_{n=1}^{\infty} \varphi_{n}^{\delta k+k-1}\left|\frac{p_{n}}{P_{n} P_{n-1}} \sum_{v=1}^{n-1}\right| b_{v}\left|P_{v}\right|^{k} \\
& \leq \sum_{n=1}^{\infty} \varphi_{n}^{\delta k+k-1} \frac{p_{n}^{k}}{P_{n}^{k} P_{n-1}} \sum_{v=1}^{n-1}\left|b_{v}\right|^{k} P_{v}^{k} / p_{v}^{k-1} \\
& =\sum_{v=1}^{\infty}\left|b_{v}\right|^{k} P_{v}^{k} / p_{v}^{k-1} \sum_{n=v+1}^{\infty} \frac{\varphi_{n}^{\delta k+k-1} p_{n}^{k}}{P_{n}^{k} P_{n-1}} \\
& =O\left\{\sum_{v=1}^{\infty} \varphi_{v}^{\delta k+k-1}\left|b_{v}\right|^{k}\right\} \\
& =O\left\{\sum_{v=1}^{\infty} \psi_{v}^{\delta k+k-1}\left|b_{v}\right|^{k}\right\} \quad \text { (by (10) and by (11)) } \\
& <\infty
\end{aligned}
$$

Now, in view of (4) we have

$$
\begin{aligned}
\sum_{n=1}^{\infty} \varphi_{n}^{\delta k+k-1}\left|C_{n, 2}\right|^{k} & =O\left\{\sum_{n=1}^{\infty} \varphi_{n}^{\delta k+k-1}\left|b_{n}\right|^{k}\right\} \\
& =O\left\{\sum_{n=1}^{\infty} \psi_{n}^{\delta k+k-1}\left|b_{n}\right|^{k}\right\} \quad(\text { by }(10)) \\
& <\infty
\end{aligned}
$$

by the assumption that $\sum a_{n}$ is summable $\psi-\left|\bar{N}, q_{n} ; \delta\right|_{k}$. This completes the proof of Theorem.

\section{References}

[1] H. Bor, On $\left|\bar{N}, p_{n}\right|_{k}$ summability factor of infinite series, Tamkang J. Math. 16 (1985), 13-20.

[2] K. N. Mishra, Absolute summability factors of type $(X, Y)$, Proc. Amer. Math. Soc. 122 (1994), 531-539.

[3] H. Seyhan, Ph. D. Thesis, Erciyes University, Kayseri, 1995.

Department of Mathematics, Erciyes University 38039, Kayseri, Turkey.

E-mail: seyhan@zirve.erciyes.edu.tr 